\title{
Physical Avatars in a Projector-Camera Tangible User Interface Enhance Quantitative Simulation Analysis and Engagement
}

\author{
Joshua Nasman \\ Rensselaer Polytechnic Institute \\ nasmajecs.rpi.edu
}

\author{
Barbara Cutler \\ Rensselaer Polytechnic Institute \\ cutler@cs.rpi.edu
}

\begin{abstract}
We present an augmented reality environment for the visualization of architectural daylighting simulations. The new visualizations focus the users' attention on the problematic aspects of a building design. Architectural design is a task particularly well suited for Tangible User Interfaces (TUIs). The user physically constructs a scale model of the building, a lighting simulation is then performed on this space, and then the simulation results are projected into the physical model by a set of calibrated projectors. A user study of an earlier version of the system revealed that users lacked accurate quantitative information about the propagation of natural light within architectural spaces and had difficulties identifying and reasoning about areas of overillumination, under-illumination, and glare. This was our motivation for two important additions to the system: physical avatar tokens within the physical scale model to specify areas of interest for glare and false color visualizations. We render viewpoints from the perspective of each avatar and indicate glare for each viewpoint. To provide users with an additional way to minimize glare and provide visual interest, we introduce new complex and interesting shading materials. These features illustrated in our tool create a more immersive and educational experience for novice and experienced designers.
\end{abstract}

\section{Introduction}

We have developed a Tangible User Interface for visualization of customized architectural daylighting simulations $[17,18]$. The user physically constructs a small scale architectural model with physical walls primitives shown in Figure 1, adding tokens to mark windows, specify surface materials, and indicate the surrounding environment (north orientation). Additionally, small avatar tokens are placed within the model to locate the typical position and usage of the space and measure the directional illumination within the simulated volume.

For each simulation, a single calibrated overhead camera captures the scene. Normally to capture 3D geometry at least two calibrated cameras are required. By having a single camera directly above the table and indicating a few fixed wall heights with differently colored top edges we are able to infer the full 3D geometry of the model. Once the 3D geometry has been captured, a closed 3D mesh is generated using the algorithm described in Cutler and Nasman [2].

Natural lighting from both the sun and the sky hemisphere is calculated for the mesh for a specified time and day and geographic location. We use a variation of "Hardware-Accelerated Global Illumination by Image Space Photon Mapping" [10] to render an image of the virtual lighting falling on each planar or curved surface in the physical model. Our simulation separates the direct light computation from the global indirect illumination which is computed using photon mapping. There are four standard sky types that are used for daylighting simulation: clear sky, intermediate sky, turbid sky and overcast sky $[12,14]$. Each of these models requires the illuminance value for the zenith [8] and measurements of the illuminance for the ground plane. For the renderings in the paper, we use the CIE clear sky model.

Our multi-projector system is run in a master slave configuration. The master sends the surface images to each process on the slave computers. Each process renders the geometry of the scene with the image textures overlaid on each wall. A common projection blending algorithm [16] takes into account the visibility and occlusions of the surfaces for each projectors and produce a smooth final illumination result on the physical model. The technique of projecting information onto a physical model is known as Spatially Augmented Reality (SAR).

The system allows users to make physical sketches with foam core walls in three heights: 5", 8", and 10". Window markers are available in two colors to create taller and shorter windows. Once a design has been completed, the user can request a time and date for simulation. In a normal interactive workflow, the user will request several times, make modifications to the room, request additional simulations, and iterate until they are satisfied with the design. We conducted a user study of the initial system and found 

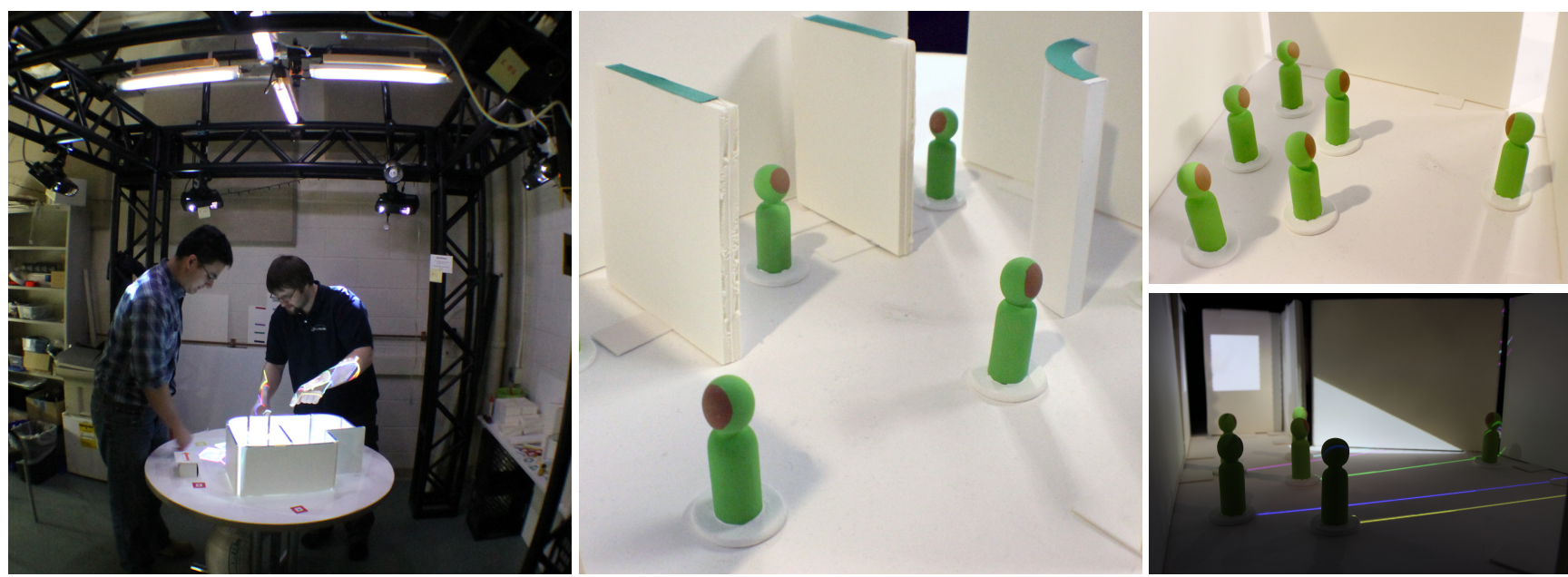

Figure 1. We provide new avatar tokens to specify the sample location and orientation of occupants in the space. The "face" of each token is detected with the overhead camera. The avatar tokens allow the designer to sketch the proposed functionality of the space. The examples above show how workers are be oriented in a cubicle office environment and how a teacher and students interact in a classroom.

that users had difficulty identifying which areas of the room were too bright, too dark, or had glare problems. Users also expressed interest in having options for more complicated window materials and lighting controls. For this reason we updated the physical controls of the system, the visualization modes, and window materials.

Our system leverages a Tangible User Interface (TUI) for interactive design, which allows quick sketching of architectural spaces. This is beneficial and convenient even for users who are familiar with standard design software tools, because the TUI provides an simpler interface to create initial rough draft models. A simple model can be created in as little as 30 seconds in our interface. In order to create a similar model in a CAD drafting tool it would take minutes instead of seconds. Importantly, it is simple to make edits to the model in response to the simulation results. Our tool provides tokens to control the most important aspects of model for daylighting: window placement and proportions, surface materials, and building orientation.

In additional to SAR there are several other options for displaying a 3D perspective of a real or virtual environments, including head mounted displays, CAVEs, etc. However, the other options do not offer the same ease of design, intuitive omni-directional display, face-to-face engagement with other users, and simultaneous visualizations for multiple virtual viewpoints. By combining these factors, we provide a powerful interface that allows the design process and lighting evaluation to happen simultaneously without the need to switch between various tools.

\subsection{Our Contributions}

The contributions we present in this paper are:

- An effective, quantitative false color visualization for use in analyzing areas of over- and under-illumination.
- Provide avatar tokens for users to place within the a physical small-scale model of a room to measure potential glare problems (negative impacts on vision due to the presence of bright light in a person's view).

- Provide the option for users to put complicated window geometry into their model while providing them with both a high detail rendering and accurate lighting information.

\section{Related Work}

Tangible User Interfaces An early TUI developed by Ishii and Ullmer [5] allowed users to manipulate digital information by controlling their system with physical icons. Jacob et al. extended the TUI by projecting information on movable physical objects [6]. The "bricks" system was the first interface to allow multiple physical controls in a TUI system to be used together to expand information shown [3]. The "JUMP" tool continued to improve controls for a TUI by using a variety of tokens in a projector-camera system to allow users to switch between multiple architectural documents to rectify them [19]. Inspired by these systems, we allow users to switch between layers of data: a daylighting rendering and a false color visualization mode of the same model. The "URban Planning" system provided an interface that enabled users to see how buildings would cast shadows on each other[20]. Our system also displays daylighting in architecture but focuses analysis on propagation and distribution of light in the interior spaces.

Projector Camera Interfaces Projector-camera systems are well suited to convey 3D information. Amano printed a normal map on paper and then used a projector camera system to project an image of the model with a light's location changing [1]. While their system only projects on a single $2 \mathrm{D}$ surface, our system projects simulation data onto a $3 \mathrm{D}$ 
model. Gartska and Peters used a Kinect to track a user's head position and orientation [4]. Based on this information, a projection of a 3D image was changed so that it appeared the user moved in relation to a $3 \mathrm{D}$ object. Menk and Koch [11] projected a simulated 3D reflected surface onto a colorless 3D model while taking into account ambient light. We also simulate light in a virtual model and project it onto a real surface but Menk and Koch were incorporating the lighting from the actual environment on a real physical geometry whereas we are running a full daylight simulation on a scale model of a space.

An early example projecting spatially immersive information on every day surfaces was The Office of the Future [15]. This work was extended in Shader Lamps[16]. Similarly to Raskar et al. [16], Sheng et al.[17, 18], and Yapo et al. [21], our system projects information on neutrally colored physical primitives to create a detailed rendering of a simulated space. Our physical primitives and rendering options provide unique extensions to their work.

In the rendering method used in our system, light is simulated bouncing through the scene through the use of photons. Photon Mapping is a method where photons are sent from a light source, allowed to bounce around a scene and then gathered at specific locations throughout the scene in order to be rendered[7]. Image space photon mapping was a GPU-accelerated version of photon mapping which allowed interactive rendering time [10]. Our renderer is based on an extension of this photon mapper which began investigating how to use the sun and sky as a light source for image spaced photon mapping [9].

\section{Motivation: Functional Architecture}

Rendering has traditionally been a field where generating an aesthetically pleasing image has higher priority than physical accuracy. In architectural design, physical accuracy is just as important as the appearance of the rendering. Knowing the locations suffering from over-illumination, under-illumination, and glare directly impacts how useful a space is to potential occupants. In spaces like art galleries, this is particularly relevant as direct sunlight can damage artwork. In a classroom, proper illumination is important both so that students can read the chalkboard, books, and laptops and so that the teacher and students can communicate effectively. Office environments need proper lighting because with workers spending 40 hours per week on detailed tasks, employers must ensure their safety and minimize fatigue and discomfort. While accurate simulation and measurement is important to creating a usable and comfortable space, it is not sufficient to create an effective design tool. Architectural daylighting design is both the process of admitting and redirecting an appropriate amount of light and making creating aesthetic choices to create comfortable, beautiful, and interesting spaces that offer healthy, productive, and inspiring work and play environments. Following these goals, we have also incorporated the simulation of intricate screens to both control the amount of light admitted through the windows and illustrate one way our interface allows architects to create beautiful interior environments.

\section{User Study of the Daylighting TUI}

We conducted a study to test users' evaluation of natural lighting in existing spaces, their ideas for renovation of the space, and the construction of a new design using our tools. To accomplish this, the participants visited an office space that has significant over- and under-illumination problems. We asked the users to make a sketch on paper and use their intuition to describe the lighting problems. After completing their initial observations, the users built a physical sketch of the space using our TUI daylighting system. The participants then used the system to evaluate lighting in the space by requesting simulations and visualizations of multiple times throughout the year and time-lapse animations. The participants then edited their physical sketch to propose a minor renovation (e.g., changing the window configuration and moving interior walls) and re-evaluated the natural lighting in the space. Finally, the participants created a new design from scratch with good daylighting principles applied. Once again they could use the system for renders and adjust as necessary.

Users appreciated the simplicity of the design process. We predicted that users would be able to sketch and modify more quickly with our design tool and that was validated by users successful designs and post-study survey comments. However, for the specific task of developing a renovation plan to improve lighting in the space, most users focused solely on the under-illumination problem and thus increased the south-facing window area in the space. Only a few of the participants noted the glare problems in the space and attempted to minimize over-illumination. When the users created their own designs they were more creative in their attempts to create functional daylighting environments, but many designs still exhibited significant areas of under- and over-illumination and users struggled to recognize the potential for glare.

While users appreciated the ability to express their design ideas and to incrementally redesign, their significant difficulties discerning which regions were functionally too bright or too dark motivated us to update the system. Renovations usually made most of the space bright enough to work, but created over-illumination in a large section of the room and created significant glare problems for the occupants. While our simulations are physically accurate, it is impossible to reproduce the brightness and contrast and high dynamic range of direct sunlight and shadow within a spatially augmented reality environment built with off-the- 

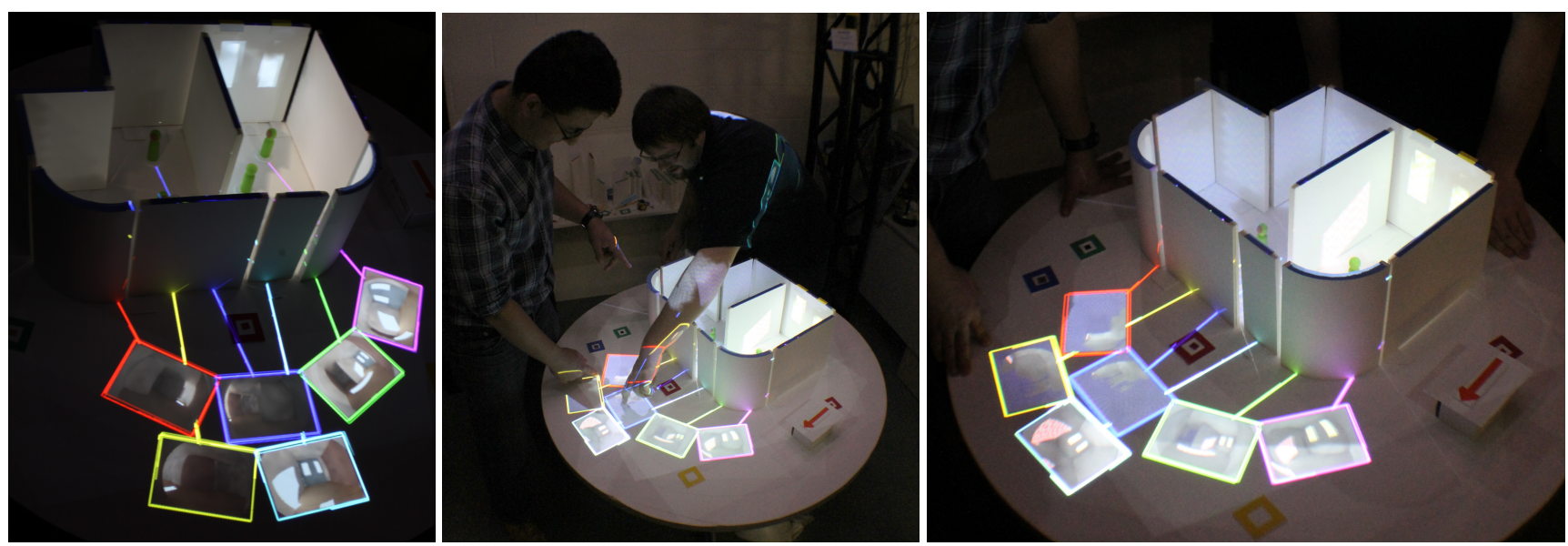

Figure 2. The most common difficulty for participants in our user study was in determining the significance of areas that were too bright or too dark. Our false color rendering mode improves this visualization challenge. Areas overlaid with a blue checkerboard are underilluminated and the red checkerboard indicates an over-illuminated area. The view point from each physical avatar are projected next to the model and also shown in Figure 3.
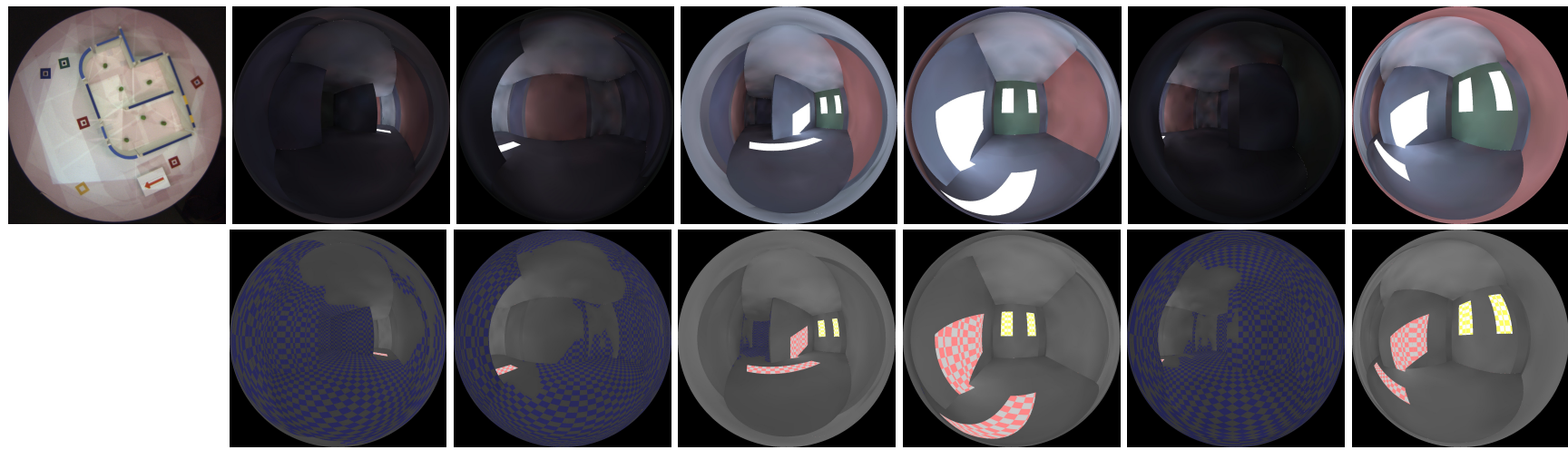

Figure 3. Fisheye views rendered from the position and orientation of each avatar token provide a visual perspective of the occupants of the design space and immerse the users in the problematic over- and under-illumination conditions of the office environment in Figure 2. Illumination problems can vary significantly across different perspectives within the same space.

shelf low dynamic range projectors. As a result, the renderings on the tabletop are a scaled representation of sunlight in the room similar to an image taken with a specified exposure.

\section{System Improvements for Effective Design}

Over-illumination and Under-illumination To communicate lighting problems more effectively, we added a false color visualization that emphasizes areas in the room that are too bright or too dark. The thresholds for over- and under-illumination are customizable. The Occupational Safety \& Health Administration (OSHA) recommends 20 to 50 foot-candles for a standard office environment [13]. We now show areas with a checkered pattern that are overilluminated (red) or under-illuminated (blue) and additionally label the view through windows in the model with a yellow checkerboard because these views often produce bright glare for the user. A checkered pattern is created by over- laying a checkerboard grid with alternating grid cells of the color above and the original illumination value in greyscale.

We believe a solid color visualization would be sufficient in many cases; however, there are situations where the visualization could be hard to evaluate. For example, in an elementary school classroom painted with bright colors, it could be very difficult to discern the a simple overlaid color visualization. Similarly, in places where there is significant over or under illumination simply scaling a color channel may produce a noticeable visualization. Thus, we choose to preserve the rendered greyscale intensity gradient in the alternate checkers so it is still possible to analyze the brightness gradient in these areas. Furthermore, this provides an opportunity for illumination to be evaluated in spaces where the color is distracting from the daylighting design task or where the problem spots in the room would be too dark or too bright for our visualization to be easily seen. In Figure 2, there is an office space that suffers from both overillumination and underillumination. We hypothesize that this 

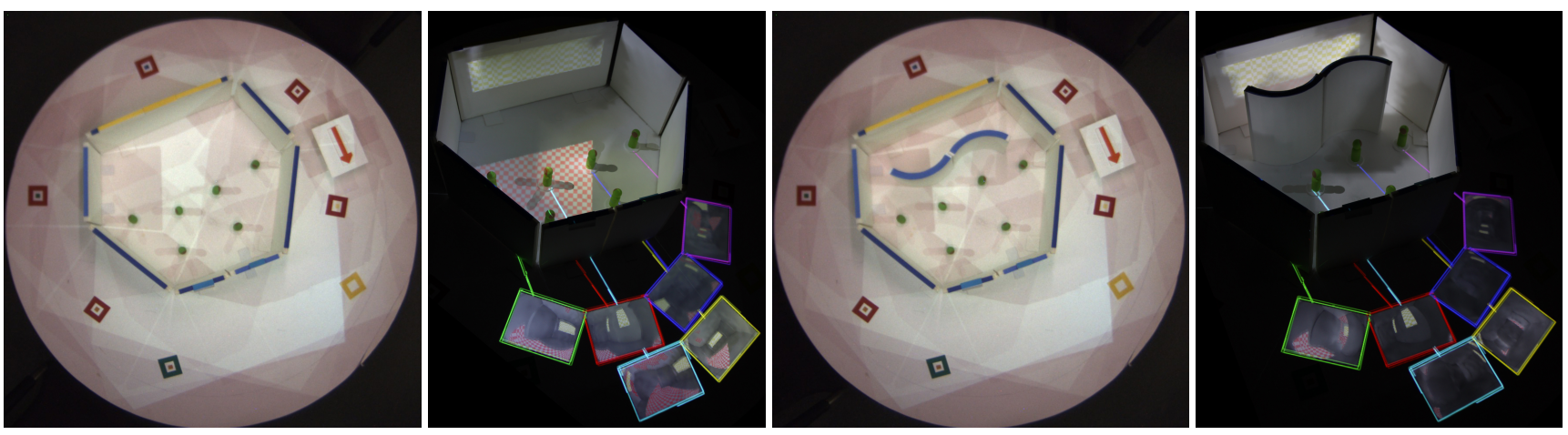

Figure 4. The left two images show an initial design proposal for an art gallery. The rendering in the room and the glare views both show significant over-illumination and glare problems. The right two images present a redesign that includes an interior curved wall to add visual interest and indirectly reflect the bright sunlight to reduce glare. The renderings of the room from each avatar are shown in Figure 5.
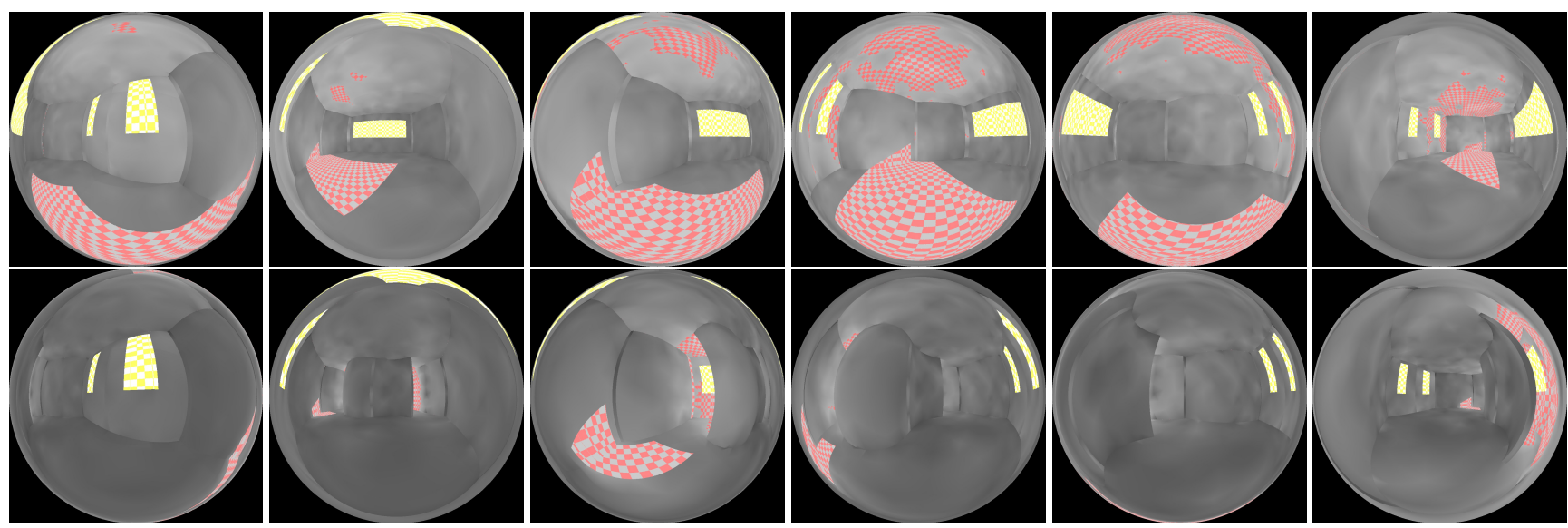

Figure 5. Fisheye views from each avatar in the gallery from Figure 4. The top row shows each view for the initial design (which includes significant glare problems). The bottom row shows the design from the same viewpoints after the re-design to reduce glare.

visualization will be of significant benefit to users of this interface. We will test this visualization in a future user study to confirm that participants are able to make better design decisions with this new quantitative information.

We chose the checkerboard pattern over other visualization techniques including using the temporal variation to convey information (e.g., a blinking visualization). We need to preserve the temporal axis for the time-lapse daily variation animation that is an important feature to visualize the dynamic lighting over the course of a day described in Sheng et al. [17, 18].

Glare Tokens In addition to over-illumination problems, we noted that many users of our system inadvertently created glare problems in their models. Only a few of the users were able to successfully increase indirect illumination using interior walls to diffusely re-direct bright sunlight from south-facing windows. We re-create a similar scenario in the example shown in Figure 4. Unfortunately, only about $25 \%$ of the participants in our original study even attempted to reduce over-illumination and glare in our system. In addition to users not being able to understand what was too bright, we believe that users had difficulties predicting and evaluating the visual perspectives the occupants would experience. To address this, we added avatar tokens to measure glare in the design. These tokens specify the position and view direction of people engaged in typical usage of the space. These tokens can be used to identify problems, and also to aid in optimization of the design for its intended function. Closeup views of these tokens are shown in Figure 1 . When viewed from the overhead camera we can detect the location of each token and also the orientation; that is, which direction the token's "face" is looking.

We render fisheye views from the each avatar's position and orientation. Because users seldom require the entire tabletop surface for their models, the renderings are automatically placed in the under-utilized areas of the table. The algorithm considers the geometry of the sketched design and how this geometry occludes the otherwise available areas for some of the projectors. Each token's rendering is connected to the associated avatar by a uniquely colored line. The user can thus design the functionality of the room and specify the location of desks in an office environment or placement of a painting in an art gallery. Based 

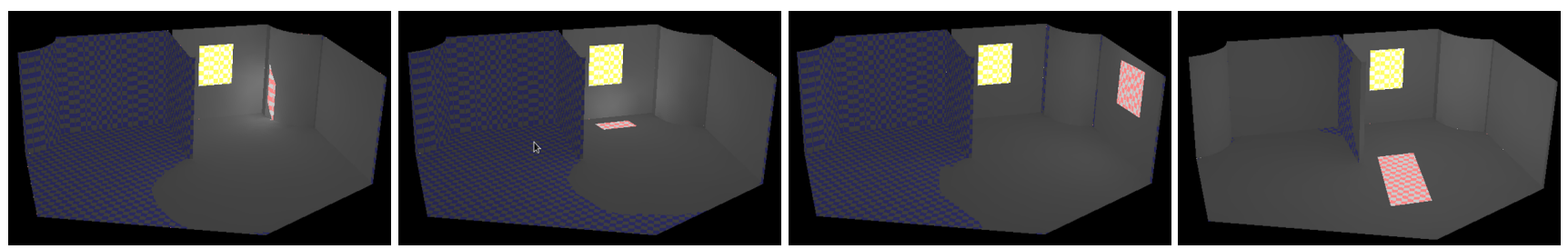

Figure 6. Over- and under-illumination are dynamic conditions and can vary drastically over a single day and throughout the year. We present simulations for June 21 at $7 \mathrm{am}$, June 21 at $11 \mathrm{am}$, December 21 at $7 \mathrm{am}$, and December 21 at 11 am. Note that in June the over-illuminated regions are near the window, while in December most of the room experience over-illumination at some point in the day.
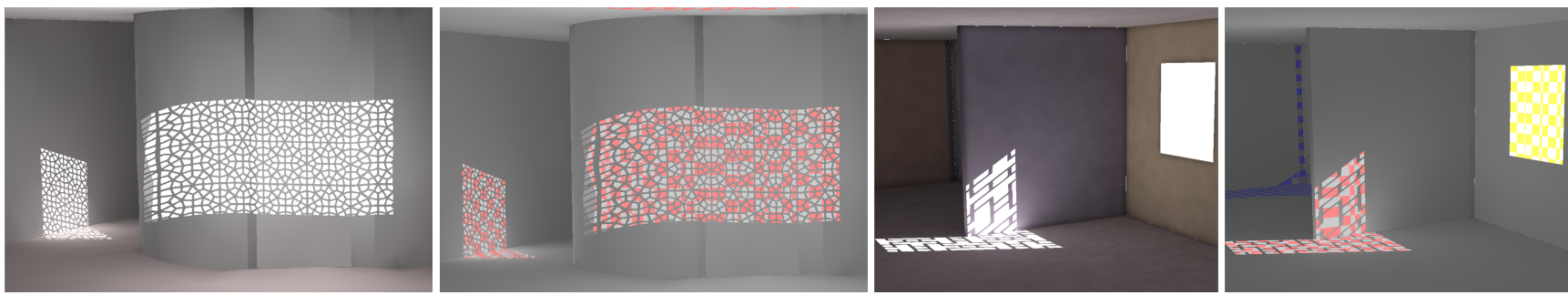

Figure 7. We added complex geometry to our window model to enable users to make the space more visually compelling. These materials allow users to reduce the amount of light streaming into the room while also making the lighting more interesting.

on these renderings, users can modify their design to correct the most significant problems. When the design modules or avatars are moved, the projector-camera system is signaled to recapture the model, recompute the lighting in the scene, and update the visualization display.

Figure 3 illustrates a wide range of variation in the lighting conditions for the occupants of an office environment similar to that used in our earlier use study. Some users will find it difficult to perform detailed task work because the space is under-illuminated while others will be hampered by overillumination and glare. The usual reaction in a space like this is to close the blinds and turn on the interior lighting. These visualizations will impress upon the the architect the need for redesign to fully leverage natural illumination. Figures 4 and 5 present an art gallery with significant glare issues before and after the placement of a large interior partition to diffusely reflect the direct sunlight. Prior to the addition, most of the space suffered from severe over-illumination. You will notice that second row has significantly lower glare for all but one token's perspective. We hypothesize that if users are given this method to directly view interior perspectives, their final designs will be appropriately tuned to the intended usage of the space.

Window Materials Users of our system had difficulty finding ways to minimize glare. While part of this is likely due to difficulties understanding illumination, part of the problem could have been a lack of sufficient tools to limit illumination. One common method to direct illumination in a space is to modify the window materials, e.g., diffusing shades, prismatic glass, or reflective light shelves. We have integrated support for complicated window screens as shown in Figure 7. These screens are one example of a window material that can both provide a way to reduce illu- mination and make the space more visually interesting. Due to the modular nature of our renderer, it will be straightforward to add many other types of materials to the interface.

\section{Discussion \& Future Work}

The modular nature of our TUI and SAR system allowed us to integrate the new false color visualization and avatar glare token features with relative ease. Extending of the detection algorithm for colored primitives on the tabletop and additions to the shader-based rendering engine are possible without the need to significantly alter the system. We will be exploring efficient simulation of a wider variety of window materials and developing an appropriate tangible interface for specifying these materials.

Our informal presentation of the new system to potential users has been promising, but our next major step will be a formal study to confirm the effectiveness of the new features of our system and to guide possible future improvements. The series of studies we have already conducted with this system has helped guide our research endeavors.

As presented in [4], head tracking could be a valuable addition to our system. Head position tracking should be relatively simple with a depth camera placed above the table facing down. Gaze recognition could be omitted as simply obtaining a view of the full rendering from the user's perspective should be sufficient. Head tracking provides several advantages. Currently, the glare views are placed around the table in areas that are most empty and visible to the projectors. Alternatively, we could place these visualizations so they are most visible to the users' current location (both location and 'up' orientation). While not providing any new information, this would remove the inconvenience of looking around the geometry or walking to the 
other side of the table to view the glare perspectives. Additionally it provides the ability to save screenshots from the user's current location. A screenshot is a view towards the center of the table from the perspective of the person's head. When a user requests a screenshot it will be saved in a session folder. This provides the user with a way to revisit lighting problems after the session has concluded and a way to collaborate. The user can send a subset of these renderings to the client or to other architects to show both potential problems with the space or to direct the client attention to certain aspects of the design.

\section{Conclusion}

Our enhancements to tabletop spatially augmented reality provide novel and interesting interaction techniques in a projector-camera system. These enhancements directly address problems revealed in a previous user study of the system. The false color visualizations in addition to the greyscale mode of rendering provide an intuitive and easy way to see when there are over- and under-illumination issues. In addition we provide a proof of concept for more complicated window designs by providing the users with screens in the windows when requested. The glare token provides an exciting new way to communicate specific visual perspectives of being in the space to the user. Finally, we propose the next enhancement for the system to further engage the users: using head tracking to target information towards the location of the user.

\section{References}

[1] T. Amano. Shading illusion: A novel way for 3-d representation on paper media. In CVPR Workshops, pages 1-6. IEEE, 2012. 2

[2] B. Cutler and J. Nasman. Interpreting physical sketches as architectural models. In C. Ceccato et al., editors, Advances in Architectural Geometry 2010, pages 15-35. Springer, 2010. 1

[3] G. W. Fitzmaurice, H. Ishii, and W. A. S. Buxton. Bricks: laying the foundations for graspable user interfaces. In $\mathrm{CHI}$ '95: Proceedings of the SIGCHI conference on Human factors in computing systems, pages 442-449, New York, NY, USA, 1995. ACM Press/Addison-Wesley Publishing Co. 2

[4] J. Garstka and G. Peters. View-dependent 3d projection using depth-image-based head tracking. 8th IEEE International Workshop on Projector Camera Systems PROCAMS, pages 52-57, 2004. 3, 6

[5] H. Ishii and B. Ullmer. Tangible bits: towards seamless interfaces between people, bits and atoms. In Proceedings of the SIGCHI conference on Human factors in computing systems, CHI '97, pages 234-241, New York, NY, USA, 1997. ACM. 2

[6] R. J. K. Jacob, H. Ishii, G. Pangaro, and J. Patten. A tangible interface for organizing information using a grid. pages 339346. ACM Press, 2001. 2
[7] H. W. Jensen. Global illumination using photon maps. In Proceedings of the eurographics workshop on Rendering techniques '96, pages 21-30, London, UK, UK, 1996. Springer-Verlag. 3

[8] M. Karayel, M. Navvab, E. Ne'eman, and S. Selkowitz. Zenith luminance and sky luminance distributions for daylighting calculations. Energy and Buildings, 6(3):283 - 291, 1984. 1

[9] E. Li. Photon mapping for architectural daylighting simulation of interior spaces. Master's thesis, Rensselaer Polytechnic Institute, December 2010. 3

[10] M. McGuire and D. Luebke. Hardware-accelerated global illumination by image space photon mapping. In Proceedings of the 2009 ACM SIGGRAPH/EuroGraphics conference on High Performance Graphics, New York, NY, USA, August 2009. ACM. 1, 3

[11] C. Menk and R. Koch. Physically-based augmentation of real objects with virtual content under the influence of ambient light. In In IEEE International Workshop on ProjectorCamera Systems, 2010. 3

[12] I. C. on Illumination. Spatial Distribution of Daylight - Luminance Distributions of Various Reference Skies. CIE technical report. CIE, 1994. 1

[13] OSHA. Osha ergonomic solutions: Computer workstations etool - workstation environment, Aug. 2010. 4

[14] R. Perez, P. Ineichen, R. Seals, J. Michalsky, and R. Stewart. Modeling daylight availability and irradiance components from direct and global irradiance. Solar energy, 44(5):271289, 1990. 1

[15] R. Raskar, G. Welch, M. Cutts, A. Lake, L. Stesin, and H. Fuchs. The office of the future: a unified approach to image-based modeling and spatially immersive displays. In Proceedings of the 25th annual conference on Computer graphics and interactive techniques, SIGGRAPH '98, pages 179-188, New York, NY, USA, 1998. ACM. 3

[16] R. Raskar, G. Welch, K.-L. Low, and D. Bandyopadhyay. Shader lamps: Animating real objects with image-based illumination. In Rendering Techniques 2001: 12th Eurographics Workshop on Rendering, pages 89-102, June 2001. 1, 3

[17] Y. Sheng, T. C. Yapo, C. Young, and B. Cutler. Virtual heliodon: Spatially augmented reality for architectural daylighting design. In $V R$, pages 63-70. IEEE, 2009. 1, 3, 5

[18] Y. Sheng, T. C. Yapo, C. Young, and B. Cutler. A spatially augmented reality sketching interface for architectural daylighting design. IEEE Transactions on Visualization and Computer Graphics, pages 38-50, January 2011. 1, 3, 5

[19] M. Terry, J. Cheung, J. Lee, T. Park, and N. Williams. Jump: a system for interactive, tangible queries of paper. In $G I$ '07: Proceedings of Graphics Interface 2007, pages 127134, New York, NY, USA, 2007. ACM. 2

[20] J. Underkoffler and H. Ishii. Urp: a luminous-tangible workbench for urban planning and design. In Proceedings of the SIGCHI conference on Human factors in computing systems. the CHI is the limit, CHI '99, pages 386-393, New York, NY, USA, 1999. ACM. 2

[21] T. Yapo, Y. Sheng, J. Nasman, A. Dolce, E. Li, and B. Cutler. Dynamic projection environments for immersive visualization. pages $1-8$, June 2010. 3 